J. Appl. Numer. Optim. 3 (2021), No. 2, pp. 263-283

Available online at http://jano.biemdas.com

https://doi.org/10.23952/jano.3.2021.2.03

\title{
A VARIATIONAL INEQUALITY FOR THE DERIVATIVE OF THE SCALAR PLAY OPERATOR
}

\author{
MARTIN BROKATE ${ }^{1,2,3, *}$, PAVEL KREJČ ${ }^{3}$ \\ ${ }^{1}$ Department of Mathematics, Technical University of Munich, Boltzmannstr. 3, Garching, 85747, Germany \\ ${ }^{2}$ Weierstrass Institute for Applied Analysis and Stochastics, Mohrenstr. 39, Berlin, 10117, Germany \\ ${ }^{3}$ Faculty of Civil Engineering, Czech Technical University in Prague, \\ Thákurova 7, 16629 Praha 6, Czech Republic
}

\begin{abstract}
We show that the directional derivative of the scalar play operator is the unique solution of a certain variational inequality. Due to the nature of the discontinuities involved, the variational inequality has an integral form based on the Kurzweil-Stieltjes integral.
\end{abstract}

Keywords. Rate independence; Variational inequality, Sweeping process; Energetic solution; Regulated function.

\section{INTRODUCTION}

The scalar stop operator $\mathscr{S}_{r}$ arises as the solution operator $\left(u, z_{0}\right) \mapsto z$ of the rate-independent evolution variational inequality

$$
\begin{array}{cl}
(\dot{u}(t)-\dot{z}(t))(z(t)-\zeta) \geq 0 \quad \forall \zeta \in Z, & \text { for a.e. } t \in(a, b), \\
z(t) \in Z \quad \forall t \in[a, b], & z(a)=z_{0},
\end{array}
$$

with $Z=[-r, r], r>0$. Its twin, the scalar play operator $\mathscr{P}_{r}$, is given by

$$
\mathscr{P}_{r}\left[u ; z_{0}\right]=u-\mathscr{S}_{r}\left[u ; z_{0}\right] .
$$

The properties of these operators have been studied extensively in, e.g., $[1,2,3,4,5,6,7,8]$.

The operators $\mathscr{S}_{r}$ and $\mathscr{P}_{r}$ are Lipschitz continuous from $W^{1,1}(a, b) \times Z$ to $W^{1,1}(a, b)$ and can be extended to Lipschitz continuous operators from $C[a, b] \times Z$ to $C[a, b]$, and even to spaces of regulated functions. However, they are not differentiable in the classical sense.

In this paper we investigate the pointwise directional derivative

$$
g(t)=\lim _{\lambda \downarrow 0} \frac{\mathscr{P}_{r}\left[u+\lambda h ; z_{0}+\lambda y_{0}\right](t)-\mathscr{P}_{r}\left[u ; z_{0}\right](t)}{\lambda}, \quad t \in[a, b],
$$

at a point $\left(u, z_{0}\right)$ in function space in the direction $\left(h, y_{0}\right)$. (The corresponding derivative for the stop is then given by $h-g$.) We have shown already in [9, Proposition 5.3] that this limit exists for $u, h \in C[a, b]$ and that it can be computed (in principle, at least) with the aid of the chain rule,

${ }^{*}$ Corresponding author.

E-mail addresses: brokate @ma.tum.de (M. Brokate), pavel.krejci@cvut.cz (P. Krejčí).

Received December 31, 2020; Accepted March 23, 2021.

(C)2021 Journal of Applied and Numerical Optimization 
applied to a certain composition of mappings involving cumulated maxima of the type $u \mapsto f$, $f(t)=\max _{\left[t_{*}, t\right]} u$ with $t_{*} \in[a, b]$.

As the main result (Theorem 2.1) of this paper, we show that $g$ is the unique solution of a certain system of variational inequalities if $u \in C[a, b]$ and $h \in C[a, b] \cap B V[a, b]$. The function $g$ turns out to belong to $B V[a, b]$ and to be one-sided continuous at each $t \in[a, b]$; however, it may be discontinuous from the right at some points and discontinuous from the left at other points even if $u$ and $h$ are smooth. (See Example 4.1 below.)

To our knowledge, this is the first result in the literature which characterizes a generalized derivative of the solution operator of a rate-independent evolution by a variational inequality. Let us add the remark that the play operator represents the solution operator of the simplest nontrivial energetic rate-independent system in the sense of [6].

Since the derivative $g$ is not absolutely continuous, it will not satisfy a variational inequality based on pointwise time derivatives like (1.1). Instead, one expects the variational inequality for $g$ to be of integral form. Such an integral form already arises when one is interested in a variational inequality formulation for the play operator itself if $u$ does not belong to $W^{1,1}(a, b)$. Indeed, for $u \in C[a, b] \cap B V[a, b]$ a proper substitute for (1.1a) is given by (see Theorem 4.1 in [4])

$$
\int_{a}^{b}(z(t)-v(t)) d w(t) \geq 0 \quad \text { for all } v \in C[a, b] \text { with values in } Z .
$$

Here $w=\mathscr{P}_{r}\left[u ; z_{0}\right]=u-z$, and the integral is a Riemann-Stieltjes integral.

When $u$ is discontinuous, an integral $\int_{a}^{b} f(t) d g(t)$ of Stieltjes type must be able to deal with functions $f$ and $g$ which are simultaneously discontinuous at certain points of $[a, b]$, in order to be useful in the present context. The Kurzweil-Stieltjes integral in particular satisfies this requirement, see Chapter 6 in [10]. Indeed, this integral was used in, e.g., [11,12] to deal with rate-independent evolutions where the "driving function" $u$ is discontinuous.

As $g$ may be discontinuous and the integral $\int_{a}^{b} g(t+) d g(t)$ appears in the variational characterization of $g$, we also use the Kurzweil-Stieltjes integral.

Another problem to be overcome is the formulation of the constraint in place of $Z$ in the variational inequality for $g$. In a general setting this constraint represents a first order conical approximation of the original constraint near the point where the derivative has to be computed. Its exact formulation and the accompanying proofs, however, are not immediately obvious and can be rather tricky, as the literature on the differentiability of solution operators for elliptic and parabolic variational inequalities shows; see, e.g., $[13,14,15,16,17,18,19,20]$.

Here, the constraint for the directional derivative $h-g$ of the stop operator, or more precisely for the right limit of $h-g$, turns out to be a time-dependent cone $K(t)$ depending on $u$ via $w$ and $z$.

In (1.1), there is no space variable, so PDE regularity problems are absent. But on the other hand, for rate-independent evolutions there is no natural Hilbert (or reflexive Banach) space framework w.r.t. the time variable. This creates problems when trying to pass to the limit in difference quotient approximations of $g$ based on (1.1). Instead, our proof of the main result is based on the decomposition of the play already used in [9] (which is not visible in the resulting variational inequality) and on piecewise constant approximations of $h$ (which, as it turns out, yield piecewise constant approximations of $g$ ). It heavily uses the well-known explicit formula for the directional derivative of the maximum functional. 
Let us close this introduction with the remark that Newton and Bouligand derivatives of the scalar play have been obtained in [21].

\section{BASIC Notions AND MAIn RESUlt}

We use the standard notations $C[a, b], B V[a, b]$ and $W^{1,1}(a, b)$ for the spaces of continuous functions, of functions of bounded variation and of absolutely continuous functions on the interval $[a, b] \subset \mathbb{R}$. We also denote by $G[a, b]$ the space of regulated functions on $[a, b]$. As usual, for a function $u:[a, b] \rightarrow \mathbb{R}$ we denote its supremum norm by $\|u\|_{\infty}$ and its variation by

$$
\operatorname{var}(u)=\sup _{\Delta} \sum_{k=1}^{N}\left|u\left(t_{k}\right)-u\left(t_{k-1}\right)\right|,
$$

the supremum being taken over all partitions $\Delta: a=t_{0}<\cdots<t_{N}=b$ of $[a, b]$.

We repeat the definition of the scalar stop from (1.1), allowing arbitrary initial values in order to simplify the formalism associated with directional derivatives. Let $Z=[-r, r]$ with $r>0$, let $\pi_{Z}: \mathbb{R} \rightarrow \mathbb{R}$ denote the projection on $Z$. It is well known that for given $\left(u, z_{0}\right) \in W^{1,1}(a, b) \times \mathbb{R}$ there exists a unique solution $z \in W^{1,1}(a, b)$ of the evolution variational inequality

$$
\begin{gathered}
(\dot{u}(t)-\dot{z}(t))(z(t)-\zeta) \geq 0 \quad \forall \zeta \in Z, \quad \text { for a.e. } t \in(a, b), \\
z(t) \in Z \quad \forall t \in[a, b], \quad z(a)=\pi_{Z}\left(z_{0}\right) .
\end{gathered}
$$

The operators $\mathscr{S}_{r}$ and $\mathscr{P}_{r}$ defined by $\mathscr{S}_{r}\left[u ; z_{0}\right]=z$ and $\mathscr{P}_{r}\left[u ; z_{0}\right]=w=u-z$ are thus welldefined on $W^{1,1}(a, b)$. It is also well known that, for $w=\mathscr{P}_{r}\left[u ; z_{0}\right]$ and $\tilde{w}=\mathscr{P}_{r}\left[\tilde{u} ; \tilde{z}_{0}\right]$,

$$
\|\tilde{w}-w\|_{\infty} \leq\|\tilde{u}-u\|_{\infty}+\left|\tilde{z}_{0}-z_{0}\right|
$$

Thus, $\mathscr{S}_{r}$ and $\mathscr{P}_{r}$ can be uniquely extended to Lipschitz continuous operators $\mathscr{S}_{r}, \mathscr{P}_{r}: C[a, b] \times$ $\mathbb{R} \rightarrow C[a, b]$, and (2.2) continues to hold for this extension.

Let $\left(u, z_{0}\right) \in C[a, b] \times \mathbb{R}$ be given, and let $z=\mathscr{S}_{r}\left[u ; z_{0}\right]$ and $w=\mathscr{P}_{r}\left[u ; z_{0}\right]$. The variational inequality for the directional derivative of $\mathscr{P}_{r}$ at $\left(u, z_{0}\right)$ involves the directional derivative of the projection $\pi_{Z}$ as well as a time-dependent restriction $K(t)$, the so-called critical cone. The sets $K(t)$ depend on $u$ by means of the behaviour of the trajectory $\{(w(t), z(t)): t \in[a, b]\}$ as follows. We set

$$
\begin{aligned}
& A_{1}=\{t \in[a, b]:|z(t)|<r\}, \\
& A_{2}^{+}=\{t \in[a, b]: z(t)=r, \exists \varepsilon>0, w=w(t) \text { on }[t, t+\varepsilon)\}, \\
& A_{2}^{-}=\{t \in[a, b]: z(t)=-r, \exists \varepsilon>0, w=w(t) \text { on }[t, t+\varepsilon)\}, \\
& A_{2}=A_{2}^{+} \cup A_{2}^{-}, \\
& A_{3}=\{t \in[a, b):|z(t)|=r, \exists \varepsilon>0, w \neq w(t) \text { on }(t, t+\varepsilon)\} .
\end{aligned}
$$

The definition of $A_{2}^{ \pm}$is to be understood as $b \in A_{2}^{ \pm}$if $z(b)= \pm r$.

Note that $[a, b]=A_{1} \cup A_{2}^{+} \cup A_{2}^{-} \cup A_{3}$ since $w$ is piecewise monotone (see, e.g., Lemma 4.4 below), and that this union is disjoint. 
We define the critical cone $K(t) \subset \mathbb{R}, t \in[a, b]$, by

$$
K(t)= \begin{cases}\mathbb{R}, & t \in A_{1}, \\ \mathbb{R}_{-}, & t \in A_{2}^{+}, \\ \mathbb{R}_{+}, & t \in A_{2}^{-}, \\ \{0\}, & t \in A_{3},\end{cases}
$$

where $\mathbb{R}_{+}=[0, \infty)$ and $\mathbb{R}_{-}=(-\infty, 0]$.

The sets $G_{K}[a, s]$ of admissible test functions associated to $K$ are defined for $s \in(a, b]$ as

$$
G_{K}[a, s]=\{v: v \in G[a, s], v(t) \in K(t) \text { for all } t \in[a, s]\} .
$$

Given $\left(h, y_{0}\right) \in G[a, b] \times \mathbb{R}$, we consider the following system of variational inequalities for an unknown function $g \in B V[a, b]$.

$$
\begin{gathered}
g(a)=h(a)-\pi_{Z}^{\prime}\left(z_{0} ; y_{0}\right), \\
h(t)-g_{+}(t) \in K(t), \quad \forall t \in[a, b], \\
\int_{a}^{s}\left(h(t)-g_{+}(t)-v(t)\right) d g(t) \geq 0, \quad \forall s \in(a, b], v \in G_{K}[a, s] .
\end{gathered}
$$

The integral in (2.6c) is a Kurzweil-Stieltjes integral, see [10]. In (2.6b) and (2.6c), $g_{+}:[a, b] \rightarrow$ $\mathbb{R}$ denotes the right limit of $g:[a, b] \rightarrow \mathbb{R}$ defined by $g_{+}(b)=g(b+)=g(b)$ and

$$
g_{+}(t)=g(t+)=\lim _{\tau \rightarrow t, \tau>t} g(\tau), \quad a \leq t<b .
$$

We also use the left limit defined by $g_{-}(a)=g(a-)=g(a)$ and

$$
g_{-}(t)=g(t-)=\lim _{\tau \rightarrow t, \tau<t} g(\tau), \quad a<t \leq b .
$$

We now state the main result of this paper.

Theorem 2.1. Let $\left(u, z_{0}\right) \in C[a, b] \times \mathbb{R}$, let $h \in C[a, b] \cap B V[a, b], y_{0} \in \mathbb{R}$. Then the pointwise directional derivative

$$
g(t)=\lim _{\lambda \downarrow 0} \frac{\mathscr{P}_{r}\left[u+\lambda h ; z_{0}+\lambda y_{0}\right](t)-\mathscr{P}_{r}\left[u ; z_{0}\right](t)}{\lambda},
$$

exists for all $t \in[a, b]$ and is the unique solution in $B V[a, b]$ of system (2.6). Moreover, $\operatorname{var}(g) \leq$ $\operatorname{var}(h)+\left|y_{0}\right|$ as well as $g(t) \in\left\{g_{+}(t), g_{-}(t)\right\}$ for all $t \in[a, b]$.

Proof. That the limit in (2.9) exists for $h \in C[a, b]$ and belongs to $B V[a, b]$ for $h \in C[a, b] \cap$ $B V[a, b]$ was proved in [9], Corollary 5.4 and Proposition 6.3. These results will also be obtained during the course of the exposition below. That $g$ solves system (2.6) and satisfies $g(t) \in\left\{g_{+}(t), g_{-}(t)\right\}$ for all $t \in[a, b]$ as well as $\operatorname{var}(g) \leq \operatorname{var}(h)+\left|y_{0}\right|$ will be proved in Propositions 5.1 and 5.2.

The proof of uniqueness follows standard lines. We present it for the sake of completeness. Let $g, \tilde{g} \in B V[a, b]$ be two solutions of (2.6), and let $s \in(a, b]$ be arbitrary. By (2.6b), the restrictions of $h-g_{+}$and of $h-\tilde{g}_{+}$to $[a, s]$ belong to $G_{K}[a, s]$. Inserting each of these functions into the variational inequality (2.6c) for the other one and adding the resulting inequalities, we obtain

$$
\int_{a}^{s}\left(\tilde{g}_{+}-g_{+}\right) d(\tilde{g}-g) \leq 0 .
$$


It follows from Proposition 6.2, applied to $\tilde{g}-g$ in place of $g$ and $[a, s]$ in place of $[a, b]$, that

$$
\frac{1}{2}\left((\tilde{g}(s)-g(s))^{2}-(\tilde{g}(a)-g(a))^{2}\right) \leq \int_{a}^{s}\left(\tilde{g}_{+}-g_{+}\right) d(\tilde{g}-g) .
$$

Since $\tilde{g}(a)=g(a)$ by (2.6a) and $s$ was arbitrary, it follows that $\tilde{g}(s)=g(s)$ for all $s>a$. This completes the proof of uniqueness.

Remark 2.1. In (2.6), we might replace the system (2.6c) w.r.t. $s$ by the single variational inequality for $s=b$; Proposition 5.2 shows that for the directional derivative $g$ this is equivalent. However, we then obtain uniqueness only in the set $B V_{r l}[a, b] \subset B V[a, b]$ of functions which are one-sided continuous (that is, $\left.v(t) \in\left\{v_{+}(t), v_{-}(t)\right\}\right)$ at every point $t$ of $[a, b]$. Indeed, in our uniqueness proof, we need not only $g$ but also $\tilde{g}$ to satisfy system (2.6c), and the one-sided continuity is crucially used in the proof of Proposition 5.2.

Our proof of the main theorem above requires a detailed study of $g$, which will be represented as the derivative of the composition of certain mappings. As this involves the chain rule, and as the chain rule does not hold in general for mappings which are only directionally differentiable, we need the slightly stronger concept of the Hadamard derivative. Let

$$
F: U \rightarrow Y, \quad U \subset X
$$

be a mapping, where $X$ and $Y$ are normed spaces, and $U \subset X$ is open. If the directional derivative $F^{\prime}(u ; h)$ exists and satisfies

$$
F^{\prime}(u ; h)=\lim _{\lambda \downarrow 0} \frac{F(u+\lambda h+r(\lambda))-F(u)}{\lambda}
$$

for all functions $r:\left[0, \lambda_{0}\right) \rightarrow X$ with $r(\lambda) / \lambda \rightarrow 0$ as $\lambda \downarrow 0, F^{\prime}(u ; h)$ is called the Hadamard derivative of $F$ at $u$ in the direction $h$. If this is true for all $h \in X, F$ is called Hadamard differentiable at $u$.

It is well known and elementary to prove that $F$ is Hadamard differentiable at $u$ if it is directionally differentiable and locally Lipschitz at $u$ and that the chain rule is valid for the composition of Hadamard differentiable functions. In the study of $g$ we will use these facts.

In this paper, we are mainly concerned with the pointwise directional derivative $g$ from (2.9). We may also view $g$ as the directional derivative of $\mathscr{P}_{r}: C[a, b] \times \mathbb{R} \rightarrow Y$ for an appropriate function space $Y$, a result which has already been obtained in [9].

Proposition 2.1. The play and the stop operator are Hadamard differentiable as mappings $\mathscr{P}_{r}, \mathscr{S}_{r}: C[a, b] \times \mathbb{R} \rightarrow L^{p}(a, b)$ for all $p \in[1, \infty)$. For $u, h \in C[a, b]$ and $z_{0}, y_{0} \in \mathbb{R}$, their Hadamard derivatives at $\left(u, z_{0}\right)$ in the direction $\left(h, y_{0}\right)$ are given by

$$
\mathscr{P}_{r}^{\prime}\left(\left[u ; z_{0}\right] ;\left[h ; y_{0}\right]\right)=g, \quad \mathscr{S}_{r}^{\prime}\left(\left[u ; z_{0}\right] ;\left[h ; y_{0}\right]\right)=h-g
$$

with $g$ from (2.9). Moreover, $\mathscr{P}_{r}\left(\left[u ; z_{0}\right] ;\left[h ; y_{0}\right]\right)$ and $\mathscr{S}_{r}\left(\left[u ; z_{0}\right] ;\left[h ; y_{0}\right]\right)$ belong to $G[a, b]$.

Proof. For $h \in C[a, b]$, the pointwise limit (2.9) exists for all $t \in[a, b]$ by Proposition 5.3 of [9], or by Proposition 4.6 below. Since

$$
\left\|\frac{\mathscr{P}_{r}\left[u+\lambda h ; z_{0}+\lambda y_{0}\right]-\mathscr{P}_{r}\left[u ; z_{0}\right]}{\lambda}\right\|_{\infty} \leq\|h\|_{\infty}+\left|y_{0}\right|,
$$

the difference quotients on the right side of (2.9) converge to $g$ in $L^{p}(a, b)$ for all $p \in[1, \infty)$ by dominated convergence. That $g \in G[a, b]$ has been proved in Proposition 6.6 of [9]. 
The mapping $\left(h, y_{0}\right) \mapsto g$ is Lipschitz continuous on $C[a, b] \times \mathbb{R}$ with respect to the supremum norm (not only with respect to the $L^{p}$ norm) in the range space.

Proposition 2.2. Let $\left(u, z_{0}\right) \in C[a, b] \times \mathbb{R}$ be given. Then

$$
\left\|\mathscr{P}_{r}^{\prime}\left(\left[u ; z_{0}\right] ;\left[\tilde{h} ; \tilde{y}_{0}\right]\right)-\mathscr{P}_{r}^{\prime}\left(\left[u ; z_{0}\right] ;\left[h ; y_{0}\right]\right)\right\|_{\infty} \leq\|\tilde{h}-h\|_{\infty}+\left|\tilde{y}_{0}-y_{0}\right|
$$

for all $\tilde{h}, h \in C[a, b]$ and all $\tilde{y}_{0}, y_{0}$ in $\mathbb{R}$.

Proof. See Proposition 4.2 below.

In view of the foregoing two propositions, we may view the mapping $\left(h, y_{0}\right) \mapsto \mathscr{P}_{r}^{\prime}\left(\left[u ; z_{0}\right] ;\left[h, y_{0}\right]\right)$ as the natural extension of the solution operator of the evolution variational inequality system (2.6) to the space $C[a, b] \times \mathbb{R}$.

\section{THE MAXIMUM FUNCTIONAL}

Let $\varphi: C[a, b] \rightarrow \mathbb{R}$ be the maximum functional

$$
\varphi(u)=\max _{t \in[a, b]} u(t), \quad u \in C[a, b],
$$

and let

$$
M(u)=\{t \in[a, b]: u(t)=\varphi(u)\}
$$

denote the set where $u$ attains its maximum. It is well-known (see, e.g., [22]) that $\varphi$ possesses a directional derivative given by

$$
\varphi^{\prime}(u ; h)=\max _{t \in M(u)} h(t)
$$

at each $u \in C[a, b]$ in any direction $h \in C[a, b]$.

Since in the proof of the main result we approximate $h$ by discontinuous functions (namely, step functions), we want to obtain a result corresponding to (3.3) for the extension of $\varphi$ to a larger space. For this, we choose the space $G[a, b]$ of regulated functions, the closure of the step functions with respect to the supremum norm. We consider

$$
\varphi(u)=\sup _{t \in[a, b]} u(t), \quad u \in G[a, b] .
$$

In order to utilize the result (3.3) on $C[a, b]$, we employ a time transformation. This is a common technique when dealing with evolutions involving discontinuous state functions, see, e.g., [2, $23,24]$.

Let $u, h \in G[a, b]$ be given. Let

$$
D \supset\{t: t \in[a, b], u \text { or } h \text { are discontinuous at } t\}
$$

be a finite or countably infinite set which includes all discontinuity points of $u$ and $h$. (The set on the right side of (3.5) has this property, see, e.g., Theorem 4.1.8 in [10].) The idea is to expand the points $t \in D$ to intervals $J_{t}$ and interpolate the values $u(t-), u(t)$ and $u(t+)$ as well as $h(t-), h(t)$ and $h(t+)$ continuously on $J_{t}$, in order to obtain continuous functions $u^{D}$ and $h^{D}$ to which (3.3) then can be applied.

We intend to define a suitable time transformation

$$
\beta: I^{D} \rightarrow I, \quad I=[a, b], \quad I^{D}=\left[a, b^{D}\right] .
$$


Let $\delta: D \rightarrow \mathbb{R}$ be a function with $\delta>0$ and $\sum_{s \in D} \delta(s)<\infty$. We define $\alpha_{+}, \alpha_{-}: I \rightarrow \mathbb{R}$ by

$$
\alpha_{-}(t)=t+\sum_{s \in D, s<t} \delta_{s}, \quad \alpha_{+}(t)=t+\sum_{s \in D, s \leq t} \delta_{s} .
$$

Then

$$
\alpha_{+}(t)=\alpha_{-}(t) \quad \Leftrightarrow \quad t \notin D
$$

We set

$$
J_{t}=\left[\alpha_{-}(t), \alpha_{+}(t)\right] .
$$

By (3.8), $J_{t}$ consists of a single point if and only if $t \notin D$.

We now set $I^{D}=\left[a, b^{D}\right]$ with $b^{D}=b+\alpha_{+}(b)$ and define the time transformation $\beta: I^{D} \rightarrow I$ by

$$
\beta(\tau)=t \quad \text { if } \tau \in J_{t}
$$

The function $\beta$ is nondecreasing, surjective, continuous and satisfies $J_{t}=\beta^{-1}(\{t\})$ for all $t \in I$.

Let $\alpha(t)$ be the midpoint of $J_{t}$,

$$
\alpha(t)=\frac{1}{2}\left(\alpha_{+}(t)+\alpha_{-}(t)\right), \quad t \in I .
$$

For any given $v \in G[a, b]$, we define $v^{D}: I^{D} \rightarrow \mathbb{R}$ by

$$
v^{D}(\alpha(t))=v(t) \quad \text { if } t \notin D,
$$

and, if $t \in D$, we define $v^{D}$ on $J_{t}$ as the linear interpolate of the values

$$
v^{D}\left(\alpha_{-}(t)\right)=v(t-), \quad v^{D}(\alpha(t))=v(t), \quad v^{D}\left(\alpha_{+}(t)\right)=v(t+) .
$$

Hence, the restrictions of $v^{D}$ to the intervals $J_{t}$ are continuous for all $t \in[a, b]$. Since $\alpha: I \rightarrow I^{D}$ is strictly monotone by construction, the mapping $v \mapsto v^{D}$ is injective.

Choosing $v=u$ resp. $v=h$ one may check that the functions $u^{D}$ and $h^{D}$ are continuous on the whole interval $I^{D}$, since all discontinuity points of $u$ and $h$ belong to $D$.

Proposition 3.1. Let $u, h \in G[a, b]$. Then $\varphi: G[a, b] \rightarrow \mathbb{R}$ is Hadamard differentiable in $u$ in the direction $h$. If in particular $u \in C[a, b]$, then

$$
\varphi^{\prime}(u ; h)=\max _{t \in M(u)} \max \{h(t-), h(t), h(t+)\}
$$

with the convention $h(a-)=h(a), h(b+)=h(b)$.

If $u$ has discontinuity points, (3.14) has to be replaced by a more complicated formula.

Proof. Let $D, u^{D}$ and $h^{D}$ be defined as above. Let $\varphi^{D}$ denote the maximum functional on $C\left(I^{D}\right)$. By construction,

$$
\varphi(u+\lambda h)=\varphi^{D}\left((u+\lambda h)^{D}\right)=\varphi^{D}\left(u^{D}+\lambda h^{D}\right)
$$

holds for every $\lambda \geq 0$. It follows that, for all $\lambda>0$

$$
\frac{\varphi(u+\lambda h)-\varphi(u)}{\lambda}=\frac{\varphi^{D}\left(u^{D}+\lambda h^{D}\right)-\varphi^{D}\left(u^{D}\right)}{\lambda} .
$$

Since $u^{D}$ and $h^{D}$ are continuous, $\left(\varphi^{D}\right)^{\prime}\left(u^{D} ; h^{D}\right)$ exists. By (3.15), the directional derivative $\varphi^{\prime}(u ; h)$ exists, too, and

$$
\left(\varphi^{D}\right)^{\prime}\left(u^{D} ; h^{D}\right)=\varphi^{\prime}(u ; h) .
$$


Applying (3.3) to $\varphi^{D}$, we obtain

$$
\varphi^{\prime}(u ; h)=\max _{\tau \in M^{D}\left(u^{D}\right)} h^{D}(\tau), \quad M^{D}\left(u^{D}\right)=\left\{\tau: u^{D}(\tau)=\varphi^{D}\left(u^{D}\right)\right\} .
$$

Since $u$ is continuous, $u^{D} \mid J_{t}$ is constant for all $t \in[a, b]$. Therefore,

$$
M^{D}\left(u^{D}\right)=\bigcup_{t \in M(u)} J_{t}, \quad \max _{\tau \in J_{t}} h^{D}(\tau)=\max \{h(t-), h(t), h(t+)\} .
$$

Now (3.14) follows from (3.16). As $\varphi$ is Lipschitz continuous on $G[a, b]$, the directional derivative is a Hadamard derivative.

In the context of the play operator, we will need to consider the maximum over subintervals of $[a, b]$. Let $J=\left[t_{*}, t^{*}\right] \subset[a, b]$. For $t \in J$, we define

$$
\begin{aligned}
F_{t}(u) & =\sup _{t_{*} \leq s \leq t} u(s), \quad u \in G[a, b], \\
M(u, t) & =\left\{\tau \in\left[t_{*}, t\right]: u(\tau)=F_{t}(u)\right\}, \quad u \in G[a, b] .
\end{aligned}
$$

Corollary 3.1. The functional $F_{t}$ is Hadamard differentiable on $G[a, b]$ for every $t \in J=\left[t_{*}, t^{*}\right] \subset$ $[a, b]$. For $u \in C[a, b]$ and $h \in G[a, b]$, its directional derivative is given by $F_{t_{*}}^{\prime}(u ; h)=h\left(t_{*}\right)$ and

$$
F_{t}^{\prime}(u ; h)=\max _{s \in M(u, t)} \hat{h}(s), \quad \hat{h}(s)= \begin{cases}\max \left\{h\left(t_{*}\right), h\left(t_{*}+\right)\right\}, & s=t_{*}, \\ \max \{h(s-), h(s), h(s+)\}, & t_{*}<s<t, \\ \max \{h(t-), h(t)\}, & s=t,\end{cases}
$$

for $t \in\left(t_{*}, t^{*}\right]$.

Proof. This is a direct consequence of Proposition 3.1 and the chain rule applied to the mapping $u \mapsto u \mid J \mapsto F_{t}(u)$.

\section{THE DIRECTIONAL DERIVATIVE OF THE SCALAR PLAY}

Let $\left(u, z_{0}\right) \in C[a, b] \times \mathbb{R}$ be given, and let $w=\mathscr{P}_{r}\left[u ; z_{0}\right]$ and $z=\mathscr{S}_{r}\left[u ; z_{0}\right]$. The trajectories $\{(u(t), w(t)): t \in[a, b]\}$ lie within the subset $\{(x, y):|x-y| \leq r\}$ of the plane $\mathbb{R}^{2}$. They consist of parts which belong to the interior, the right or the left boundary of this subset. Accordingly, the time interval $[a, b]$ decomposes into the three disjoint sets

$$
\begin{aligned}
I_{0} & =\{t \in[a, b]:|u(t)-w(t)|=|z(t)|<r\}, \\
I_{+} & =\{t \in[a, b]: u(t)-w(t)=z(t)=r\}, \\
I_{-} & =\{t \in[a, b]: u(t)-w(t)=z(t)=-r\} .
\end{aligned}
$$

We also define

$$
I_{\partial}=I_{+} \cup I_{-} .
$$

The set $I_{0}$ is a relatively open subset of $[a, b]$, the sets $I_{ \pm}$and $I_{\partial}$ are compact.

A closed interval $J \subset[a, b]$ is called a plus interval for $\left(u, z_{0}\right)$ if $J \subset I_{+} \cup I_{0}$ and $J \cap I_{+} \neq \emptyset$; it is called a minus interval for $\left(u, z_{0}\right)$ if $J \subset I_{-} \cup I_{0}$ and $J \cap I_{-} \neq \emptyset$. Thus, on a plus interval the trajectory $\{(u(t), w(t))\}$ hits the right but not the left boundary of the admissible domain $\{(x, y):|x-y| \leq r\}$, and vice versa on a minus interval.

A partition $\Delta: a=\tau_{0}<\ldots<\tau_{R}=b$ is called regular for $\left(u, z_{0}\right)$ if $z\left(\tau_{i}\right)=0$ for all $0<i<R$ and if all intervals $\left[\tau_{i-1}, \tau_{i}\right]$ are plus or minus intervals for $\left(u, z_{0}\right)$. 
If $I_{0}=[a, b]$, that is, if the trajectory never touches the boundary, then $w=u-z$ is constant on $[a, b]$ and $\left|z_{0}\right|<r$. Since this continues to hold if we vary $\left(u, z_{0}\right)$ by a sufficiently small amount,

$$
\mathscr{P}_{r}\left[\tilde{u} ; \tilde{z}_{0}\right](t)=\tilde{u}(a)-\tilde{z}_{0}, \quad t \in[a, b],
$$

if $\left(\tilde{u}, \tilde{z}_{0}\right)$ is sufficiently close to $\left(u, z_{0}\right)$. Consequently, at $\left(u, z_{0}\right)$ the play $\mathscr{P}_{r}: C[a, b] \times \mathbb{R} \rightarrow$ $C[a, b]$ has the Fréchet derivative $\mathscr{P}_{r}^{\prime}\left[u ; z_{0}\right]$ given by

$$
\mathscr{P}_{r}^{\prime}\left[u ; z_{0}\right]\left(h, y_{0}\right)(t)=h(a)-y_{0}, \quad t \in[a, b] .
$$

Since $\mathscr{S}_{r}\left[u ; z_{0}\right]+\mathscr{P}_{r}\left[u ; z_{0}\right]=u$,

$$
\mathscr{S}_{r}^{\prime}\left[u ; z_{0}\right]\left(h, y_{0}\right)(t)=h(t)-h(a)+y_{0}, \quad t \in[a, b] .
$$

Thus, we only have to study the case $[a, b] \neq I_{0}$.

Lemma 4.1. Let $\left(u, z_{0}\right) \in C[a, b] \times \mathbb{R}$, let $[a, b] \neq I_{0}$. Then there exists a regular partition for $\left(u, z_{0}\right)$.

Proof. Starting from $\Delta=\{a, b\}$, we successively add points $t \in(a, b)$ with $z(t)=0$ as follows until we arrive at a regular partition. Let $\delta>0$ be such that $|z(t)-z(s)|<r$ for all $s, t \in[a, b]$ with $|s-t| \leq \delta$. Let $\Delta=\left\{\tau_{i}\right\}$ be a partition of $[a, b]$ such that every interval $\left[\tau_{i-1}, \tau_{i}\right]$ includes at least one point from $I_{\partial}$ and that $z\left(\tau_{i}\right)=0$ at all partition points in $(a, b)$. (The partition $\Delta=\{a, b\}$ has this property since we assumed that $[a, b] \neq I_{0}$.) If $\Delta$ is not regular, there exists an interval $J=\left[\tau_{k-1}, \tau_{k}\right]$, which includes points $s, t$ with $z(s)=-r$ and $z(t)=r$. We choose $\tau^{\prime}$ between $s$ and $t$ with $z\left(\tau^{\prime}\right)=0$. All partition intervals of $\Delta^{\prime}=\Delta \cup\left\{\tau^{\prime}\right\}$ then include at least one point from $I_{\partial}$. Since the distance of the new point $\tau^{\prime}$ from $s$ and $t$, and thus from all partition points of $\Delta$, is at least $\delta$, this process comes to an end after a finite number of steps. The resulting partition is regular.

Lemma 4.2. Let $\left(u, z_{0}\right) \in C[a, b] \times \mathbb{R}$. We have

$$
\mathscr{P}_{r}\left[-u ;-z_{0}\right]=-\mathscr{P}_{r}\left[u ; z_{0}\right] .
$$

If $J$ is a minus interval for $\left(u, z_{0}\right)$, then $J$ is a plus interval for $\left(-u,-z_{0}\right)$. If $\Delta$ is a regular partition for $\left(u, z_{0}\right)$, then $\Delta$ is a regular partition for $\left(-u,-z_{0}\right)$.

Proof. The equality (4.5) is an elementary property of the play and can be derived, e.g., via (2.1). The other assertions follow directly from (4.5).

Lemma 4.3. Let $J=\left[t_{*}, t^{*}\right]$ be a plus resp. a minus interval for $\left(u, z_{0}\right) \in C[a, b] \times \mathbb{R}$. Then $w=\mathscr{P}_{r}\left[u ; z_{0}\right]$ satisfies

$$
\begin{array}{ll}
w(t)=\max \left\{w\left(t_{*}\right), \max _{t_{*} \leq s \leq t} u(s)-r\right\}, & \text { for all } t \in J, \\
w(t)=\min \left\{w\left(t_{*}\right), \min _{t_{*} \leq s \leq t} u(s)+r\right\}, & \text { for all } t \in J,
\end{array}
$$

respectively. In particular, $w$ is nondecreasing resp. nonincreasing on $J$.

Proof. The first equality was proved in [9, Lemma 5.1]. The second follows from the first, using Lemma 4.2. 
For a given pair $\left(u, z_{0}\right) \in C[a, b] \times \mathbb{R}$, let

$$
U_{\delta}=\left\{\left(\tilde{u}, \tilde{z}_{0}\right) \in G[a, b] \times \mathbb{R}:\|\tilde{u}-u\|_{\infty}<\delta,\left|\tilde{z}_{0}-z_{0}\right|<\delta\right\}
$$

be its $\delta$-neighbourhood in $G[a, b] \times \mathbb{R}$, where $\delta>0$. Let $\left(\tilde{u}, \tilde{z}_{0}\right) \in U_{\delta}$, and let $D \subset[a, b]$ include all discontinuity points of $\tilde{u}$. With $\alpha^{D}=\alpha$ and $\tilde{u}^{D}$ as in (3.11)-(3.13) we define $\tilde{w}:[a, b] \rightarrow \mathbb{R}$ by

$$
\tilde{w}(t)=\tilde{w}^{D}\left(\alpha^{D}(t)\right), \quad \tilde{w}^{D}=\mathscr{P}_{r}\left[\tilde{u}^{D} ; \tilde{z}_{0}\right] .
$$

Lemma 4.4. Let $\Delta$ be a regular partition for $\left(u, z_{0}\right) \in C[a, b] \times \mathbb{R}$. Then there exists $\delta>0$ such that, for all $\left(\tilde{u}, \tilde{z}_{0}\right) \in U_{\delta}$ from (4.7) and all plus resp. minus partition intervals $J=\left[t_{*}, t^{*}\right]$ of $\Delta$, the function $\tilde{w}$ defined in (4.8) satisfies

$$
\begin{array}{ll}
\tilde{w}(t)=\max \left\{\tilde{w}\left(t_{*}\right), \sup _{t_{*} \leq s \leq t} \tilde{u}(s)-r\right\}, & \text { for all } t \in J, \\
\tilde{w}(t)=\min \left\{\tilde{w}\left(t_{*}\right), \inf _{t_{*} \leq s \leq t} \tilde{u}(s)+r\right\}, & \text { for all } t \in J,
\end{array}
$$

respectively. In particular, $\tilde{w}$ is piecewise monotone on $[a, b]$. Moreover, the mapping $\left(\tilde{u}, \tilde{z}_{0}\right) \mapsto$ $\tilde{w}$ is Lipschitz continuous on $U_{\delta}$,

$$
\left\|\tilde{w}_{2}-\tilde{w}_{1}\right\|_{\infty} \leq\left\|\tilde{u}_{2}-\tilde{u}_{1}\right\|_{\infty}+\left|\tilde{z}_{0,2}-\tilde{z}_{0,1}\right| .
$$

Proof. Let $J=\left[t_{*}, t^{*}\right]$ be a plus interval for $\left(u, z_{0}\right)$, so $\min _{J} z+r>0$. Let $\left(\tilde{u}, \tilde{z}_{0}\right) \in G[a, b] \times \mathbb{R}$. let $D \subset[a, b]$ include all discontinuity points of $\tilde{u}$, and let $J^{D}=\left[\alpha^{D}\left(t_{*}\right), \alpha^{D}\left(t^{*}\right)\right]$. By construction, $\tilde{z}^{D}=\mathscr{S}_{r}\left[\tilde{u}^{D} ; \tilde{z_{0}}\right]$ satisfies $\min _{J^{D}} \tilde{z}^{D}=\min _{J} \tilde{z}$. Since $\mathscr{S}_{r}$ is Lipschitz continuous on $C[a, b] \times \mathbb{R}$, there exists $\delta_{J}>0$ such that $\min _{J} \tilde{z}+r>0$ if $\|\tilde{u}-u\|_{\infty}<\delta_{J}$ and $\left|\tilde{z}_{0}-z_{0}\right|<\delta_{J}$. For every such pair $\left(\tilde{u}, \tilde{z}_{0}\right), J^{D}$ is thus a plus interval for $\left(\tilde{u}^{D}, \tilde{z}_{0}\right)$. From Lemma 4.3, applied to $\left(\tilde{u}^{D}, \tilde{z}_{0}\right)$ on $J^{D}$, it follows that

$$
\begin{aligned}
\tilde{w}(t) & =\tilde{w}^{D}\left(\alpha^{D}(t)\right)=\max \left\{\tilde{w}^{D}\left(\alpha^{D}\left(t_{*}\right)\right), \max _{\alpha^{D}\left(t_{*}\right) \leq \sigma \leq \alpha^{D}(t)} \tilde{u}^{D}(\sigma)-r\right\} \\
& =\max \left\{\tilde{w}\left(t_{*}\right), \sup _{t_{*} \leq s \leq t} \tilde{u}(s)-r\right\} .
\end{aligned}
$$

If $J$ is a minus interval, an analogous argument applies. Taking $\delta=\min \delta_{J}$ over all subintervals $J$ of $\Delta$ yields (4.9). To prove (4.10), one checks by induction over the subintervals of $\Delta$, using (4.9), that $\left|\tilde{w}_{2}(t)-\tilde{w}_{1}(t)\right|$ is bounded by the right side of (4.10) for all $t \in[a, b]$.

By Lemma 4.4, for given $\left(u, z_{0}\right) \in C[a, b] \times \mathbb{R}$

$$
\mathscr{P}_{r, t}\left[\tilde{u} ; \tilde{z}_{0}\right]=\tilde{w}(t)
$$

defines operators

$$
\mathscr{P}_{r, t}: U_{\delta} \rightarrow \mathbb{R}, \quad t \in[a, b],
$$

for sufficiently small $\delta>0$. By Lemmas 4.3 and 4.4, we have that

$$
\mathscr{P}_{r, t}\left[\tilde{u} ; \tilde{z}_{0}\right]=\mathscr{P}_{r}\left[\tilde{u} ; \tilde{z}_{0}\right](t), \quad t \in[a, b],
$$

holds for all pairs $\left(\tilde{u}, \tilde{z}_{0}\right) \in U_{\delta} \cap(C[a, b] \times \mathbb{R})$. Extending $\mathscr{P}_{r, t}$ to $-U_{\delta}$ by

$$
\mathscr{P}_{r, t}\left[\tilde{u} ; \tilde{z}_{0}\right]=-\mathscr{P}_{r, t}\left[-\tilde{u} ;-\tilde{z}_{0}\right]
$$

we see from Lemma 4.2 that (4.13) holds on $\left(-U_{\delta}\right) \cap(C[a, b] \times \mathbb{R})$, too.

We derive a formula for the directional derivative of $\mathscr{P}_{r, t}$ at $\left(u, z_{0}\right)$. For $t=a$,

$$
\mathscr{P}_{r, a}\left[u ; z_{0}\right]=u(a)-\pi_{Z}\left(z_{0}\right)
$$


so $\mathscr{P}_{r, a}$ is Hadamard differentiable at $\left(u, z_{0}\right)$ and

$$
\mathscr{P}_{r, a}^{\prime}\left(\left[u ; z_{0}\right] ;\left[h ; y_{0}\right]\right)=h(a)-\pi_{Z}^{\prime}\left(z_{0} ; y_{0}\right)
$$

holds for all $\left(h, y_{0}\right) \in G[a, b] \times \mathbb{R}$.

Proposition 4.1. Let $J=\left[t_{*}, t^{*}\right]$ be a plus interval for $\left(u, z_{0}\right) \in C[a, b] \times \mathbb{R}$, let $\mathscr{P}_{r, t_{*}}$ be Hadamard differentiable at $\left(u, z_{0}\right)$. Then $\mathscr{P}_{r, t}$ is Hadamard differentiable at $\left(u, z_{0}\right)$ for all $t \in J$. The derivative

$$
g(t)=\mathscr{P}_{r, t}^{\prime}\left(\left[u ; z_{0}\right] ;\left[h ; y_{0}\right]\right), \quad t \in J,
$$

in the direction $\left(h, y_{0}\right) \in G[a, b] \times \mathbb{R}$ satisfies, for all $t \in\left(t_{*}, t^{*}\right]$,

$$
g(t)= \begin{cases}g\left(t_{*}\right), & w\left(t_{*}\right)>F_{t}(u)-r, \\ \max \left\{g\left(t_{*}\right), F_{t}^{\prime}(u ; h)\right\}, & w\left(t_{*}\right)=F_{t}(u)-r, \\ F_{t}^{\prime}(u ; h), & w\left(t_{*}\right)<F_{t}(u)-r,\end{cases}
$$

where $F_{t}^{\prime}(u ; h)$ is given in (3.18).

Proof. The maximum $f: \mathbb{R}^{2} \rightarrow \mathbb{R}, f(x, y)=\max \{x, y\}$, is Hadamard differentiable and has the directional derivative

$$
f^{\prime}((x, y) ;(\xi, \eta))= \begin{cases}\xi, & y<x, \\ \max \{\xi, \eta\}, & y=x, \\ \eta, & y>x .\end{cases}
$$

By (4.11) and Lemma 4.4, for $\left(\tilde{u}, \tilde{z}_{0}\right) \in U_{\delta}$, we have

$$
\mathscr{P}_{r, t}\left[\tilde{u} ; \tilde{z}_{0}\right]=\max \left\{\mathscr{P}_{r, t_{*}}\left[\tilde{u} ; \tilde{z}_{0}\right], F_{t}(\tilde{u})-r\right\} \text {. }
$$

Therefore, all assertions follow from the chain rule and Corollary 3.1.

Proposition 4.2. Let $\left(u, z_{0}\right) \in C[a, b] \times \mathbb{R}$. Then $\mathscr{P}_{r, t}: U_{\delta} \cup\left(-U_{\delta}\right) \rightarrow \mathbb{R}$ is Hadamard differentiable at $\left(u, z_{0}\right)$ and $\left(-u,-z_{0}\right)$ for all $t \in[a, b]$ if $\delta>0$ is sufficiently small, and

$$
\mathscr{P}_{r, t}^{\prime}\left(\left[u ; z_{0}\right] ;\left[h, y_{0}\right]\right)=-\mathscr{P}_{r, t}^{\prime}\left(\left[-u ;-z_{0}\right] ;\left[-h,-y_{0}\right]\right)
$$

for all $h \in G[a, b], y_{0} \in \mathbb{R}$. Moreover, setting

$$
\tilde{g}(t)=\mathscr{P}_{r, t}^{\prime}\left(\left[u ; z_{0}\right] ;\left[\tilde{h}, \tilde{y}_{0}\right]\right), \quad g(t)=\mathscr{P}_{r, t}^{\prime}\left(\left[u ; z_{0}\right] ;\left[h, y_{0}\right]\right),
$$

we have that

$$
\sup _{t \in[a, b]}\|\tilde{g}-g\|_{\infty} \leq\|\tilde{h}-h\|_{\infty}+\left|\tilde{y}_{0}-y_{0}\right|
$$

for all $\tilde{h}, h \in G[a, b]$ and all $\tilde{y}_{0}, y_{0} \in \mathbb{R}$.

Proof. We first prove (4.19). By (4.14), $\mathscr{P}_{r, t}$ is defined on $U_{\delta} \cup\left(-U_{\delta}\right)$ for $\delta>0$ small enough and satisfies $\mathscr{P}_{r, t}\left[\tilde{u} ; \tilde{z}_{0}\right]=-\mathscr{P}_{r, t}\left[-\tilde{u} ;-\tilde{z}_{0}\right]$ on $U_{\delta}$. Passing to the limit in the difference quotients corresponding to (4.19) shows that if, for given $\left(t, h, y_{0}\right)$, one of the derivatives appearing in (4.19) exists, then the other exists too, and (4.19) holds.

For $t=a$, we refer to (4.15) and (4.16). On $(a, b]$, we use induction over the successive subintervals of a regular partition $\Delta$ for $\left(u, z_{0}\right)$. Let $J=\left[t_{*}, t^{*}\right]$ be such a subinterval. By the induction hypothesis, $\mathscr{P}_{r, t_{*}}$ is Hadamard differentiable at $\left(u, z_{0}\right)$, and hence also at $\left(-u,-z_{0}\right)$. If $J$ is a plus interval for $\left(u, z_{0}\right)$, we apply Proposition 4.1. It follows that $\mathscr{P}_{r, t}$ is Hadamard differentiable at $\left(u, z_{0}\right)$, and hence also at $\left(-u,-z_{0}\right)$, for all $t \in\left(t_{*}, t^{*}\right]$. If $J$ is a minus interval for 
$\left(u, z_{0}\right)$, it is a plus interval for $\left(-u,-z_{0}\right)$ by Lemma 4.2. By Proposition 4.1, $\mathscr{P}_{r, t}$ is Hadamard differentiable at $\left(-u,-z_{0}\right)$, and hence at $\left(u, z_{0}\right)$, for all $t \in\left(t_{*}, t^{*}\right]$. This completes the induction step and thus the proof of (4.19).

That (4.20) holds follows from (4.10) applied to the difference quotients which define $\tilde{g}$ and $g$.

The pointwise directional derivative $g$ can be discontinuous from the right or from the left at certain points of $[a, b]$ even if $u$ and $h$ are smooth, as the following example shows.

Example 4.1. Let $[a, b]=[0,4], z_{0}=r$ and $y_{0}=0$. Let $u:[0,4] \rightarrow \mathbb{R}$ be a smooth function which satisfies

$$
\begin{gathered}
r=u(0)=u(1)<u(2)=u(3)<u(4) \\
0 \leq u<r \quad \text { on }(0,1) \\
u(2)-r \leq u<u(2) \quad \text { on }(2,3) \\
u^{\prime}>0 \quad \text { on }(1,2) \text { and on }(3,4) .
\end{gathered}
$$

Then $[0,4]$ is a plus interval for $\left(u, z_{0}\right)$ and $w=\mathscr{P}_{r}\left[u ; z_{0}\right]$ coincides with the cumulated maximum

$$
w(t)=F_{t}(u)-r=\max _{0 \leq s \leq t} u(s)-r, \quad t \in[0,4] .
$$

The sets $M(u, t)$ from (3.17) become

$$
M(u, t)=\left\{\begin{array}{ll}
\{0\}, & t \in[0,1), \\
\{0,1\}, & t=1, \\
\{t\}, & t \in(1,2),
\end{array} \quad M(u, t)= \begin{cases}\{2\}, & t \in[2,3), \\
\{2,3\}, & t=3, \\
\{t\}, & t \in(3,4) .\end{cases}\right.
$$

For every $h \in C[0,4]$, we obtain from Corollary 3.1 and Proposition 4.1 that the one-sided limits of $g$ satisfy

$$
\begin{array}{lll}
g(1-)=h(0), & g(1)=\max \{h(0), h(1)\}, & g(1+)=h(1), \\
g(3-)=h(2), & g(3)=\max \{h(2), h(3)\}, & g(3+)=h(3) .
\end{array}
$$

Thus, all combinations of discontinuity behavior of $g$ at $t=1$ and $t=3$ can be achieved by suitable choices of an arbitrarily smooth variation $h$.

\section{THE VARIATIONAL INEQUALITY FOR THE DERIVATIVE}

We want to derive a variational inequality for the function $g:[a, b] \rightarrow \mathbb{R}$,

$$
g(t)=\mathscr{P}_{r, t}^{\prime}\left(\left[u ; z_{0}\right] ;\left[h, y_{0}\right]\right)=\lim _{\lambda \downarrow 0} \frac{\mathscr{P}_{r, t}\left[u+\lambda h ; z_{0}+\lambda y_{0}\right]-\mathscr{P}_{r, t}\left[u ; z_{0}\right]}{\lambda} .
$$

In this section, we generally assume that $\left(u, z_{0}\right) \in C[a, b] \times \mathbb{R}$ as well as $\left(h, y_{0}\right) \in G[a, b] \times \mathbb{R}$ are given and that $\Delta$ is a regular partition for $\left(u, z_{0}\right)$. As before, we set $w=\mathscr{P}_{r}\left[u ; z_{0}\right]$ and $z=\mathscr{S}_{r}\left[u ; z_{0}\right]$.

We first investigate the behaviour of $g$ on a plus interval. In the following development up to Lemma 5.8, we assume that $J=\left[t_{*}, t^{*}\right]$ is a given plus interval for $\left(u, z_{0}\right)$.

We look at intervals where the trajectory $\{(u(t), w(t))\}$ does not touch the boundary of the admissible region $\{(x, y):|x-y| \leq r\}$. 
Lemma 5.1. The function $g$ is constant on every interval contained in $J \cap I_{0}$.

Proof. Let $K=\left[\tau_{*}, \tau^{*}\right] \subset J \cap I_{0}$. Since $w=\mathscr{P}_{r}\left[u ; z_{0}\right]$ is locally constant on $I_{0}$, according to (5.1), (4.11) and Lemma 4.4, it suffices to show that the function

$$
\tilde{w}(t)=\max \left\{\tilde{w}\left(t_{*}\right), \sup _{t_{*} \leq s \leq t} \tilde{u}(s)-r\right\}
$$

is constant on $K$ if $\left(\tilde{u}, \tilde{z}_{0}\right)$ is sufficiently close to $\left(u, z_{0}\right)$.

Let us assume that $\tilde{w}$ is not constant on $K$. As $\tilde{w}$ is nondecreasing on $J$, we must have $\tilde{w}\left(\tau^{*}\right)>\tilde{w}\left(\tau_{*}\right)$. It follows from (5.2) that

$$
\sup _{s \in K} \tilde{w}(s)=\tilde{w}\left(\tau^{*}\right)=\sup _{s \in K} \tilde{u}(s)-r .
$$

Now

$$
r=\sup _{K} \tilde{u}-\sup _{K} \tilde{w} \leq \sup _{K}(\tilde{u}-\tilde{w}) \leq \sup _{K}(u-w)+\|\tilde{u}-u\|_{\infty}+\|\tilde{w}-w\|_{\infty} .
$$

Since $K \subset I_{0}$, we have $\sup _{K}(u-w)<r$. Thus, if $\left(\tilde{u}, \tilde{z}_{0}\right)$ is sufficiently close to $\left(u, z_{0}\right)$, a contradiction arises due to (4.10).

If $K$ is half-open or open, interior approximation by closed intervals yields the assertion.

We recall the definitions ( $u$ is continuous)

$$
F_{t}(u)=\max _{t_{*} \leq s \leq t} u(s), \quad M(u, t)=\left\{\tau \in\left[t_{*}, t\right]: u(\tau)=F_{t}(u)\right\} .
$$

Since $t \mapsto F_{t}(u)$ is nondecreasing, we have for all $s, t \in J$ with $t<s$

$$
M(u, t) \subset M(u, s) \quad \text { or } \quad M(u, t)<M(u, s)
$$

(to be understood pointwise, $\tau<\sigma$ for all $\tau \in M(u, t)$ and all $\sigma \in M(u, s)$ ).

Next, we investigate the behaviour of $g$ at points $t \notin I_{0}$. We denote by

$$
t_{+}=\min \left(J \cap I_{+}\right)
$$

the first point in $J$ where the trajectory $\{u(t), w(t))\}$ touches the right boundary of $\{(x, y)$ : $|x-y| \leq r\}$.

Lemma 5.2. We have $w\left(t_{*}\right)=w\left(t_{+}\right)=u\left(t_{+}\right)-r,\left[t_{*}, t_{+}\right) \subset I_{0}, M\left(u, t_{+}\right)=\left\{t_{+}\right\}$and $M(u, t) \subset$ $\left[t_{+}, t\right]$ for all $t \in\left[t_{+}, t^{*}\right]$.

Proof. By definition of $t_{+}$, we have $w\left(t_{+}\right)=u\left(t_{+}\right)-r$ and $\left[t_{*}, t_{+}\right) \subset I_{0}$. Since $w$ is locally constant in $I_{0}$, it follows that $u-r<w=u\left(t_{+}\right)-r$ on $\left[t_{*}, t_{+}\right)$. Thus $M\left(u, t_{+}\right)=\left\{t_{+}\right\}$. From (5.4), it follows that $M(u, t) \subset\left[t_{+}, t\right]$ if $t \geq t_{+}$.

The value of $g$ at a boundary point $t$ depends on $h(t)$ and on the left limits of $g$ and $h$ at $t$.

Lemma 5.3. Let $t_{*}<t \in J \cap I_{+}$. Then $g(t-)$ exists and

$$
g(t)=\max \{h(t), h(t-), g(t-)\} .
$$

More precisely, for $t>t_{+}$three cases arise.

(i) Let $u<u(t)$ on $\left[t_{+}, t\right)$. Then

$$
g(t)=\max \{h(t), h(t-)\}, \quad g(t-)=h(t-) .
$$

(ii) Iffor some $\hat{t} \in\left[t_{+}, t\right)$ we have $u(\hat{t})=u(t)$ and $u<u(t)$ on $(\hat{t}, t)$, then

$$
g(t)=\max \{h(t), h(t-), g(t-)\}, \quad g(t-)=g(\hat{t}) .
$$


(iii) Let $u\left(\tau_{k}\right)=u(t)$ for some sequence $\tau_{k} \uparrow t$ with $\tau_{k}<t$ for all $k$. Then

$$
g(t)=\max \{h(t), g(t-)\}, \quad g(t-) \geq h(t-) .
$$

Proof. For $t_{*}<t=t_{+}$we have $g\left(t_{*}\right)=g(t-)$ by Lemma 5.1. From Lemma 5.2, it follows that $M(u, t)=\{t\}$ and that the second case in (4.18) arises. From Corollary 3.1, we obtain $F_{t}^{\prime}(u ; h)=\max \{h(t-), h(t)\}$. Thus (5.6) holds at $t=t_{+}$.

Let now $t>t_{+}$. We have $u \leq u(t)$ on some interval $(t-\varepsilon, t)$, since otherwise

$$
u(\tau)-w(\tau) \geq u(\tau)-w(t)>u(t)-w(t)=r
$$

for some $\tau<t$, which is impossible. Therefore, for $t>t_{+}$no other cases arise besides (i)-(iii).

(i) We have $g(t)=F_{t}^{\prime}(u ; h)=\max \{h(t-), h(t)\}$ since $M(u, t)=\{t\}$ and $w\left(t_{*}\right)=u\left(t_{+}\right)-r<$ $u(t)-r$. We define

$$
\tau_{k}=\min \{s: s \in[a, t], u(s)=u(t)-1 / k\}, \quad k>1 /\left(u(t)-u\left(t_{+}\right)\right) .
$$

Then $M(u, \tau) \subset\left[\tau_{k}, t\right)$ for all $\tau \in\left[\tau_{k}, t\right)$. Since $\tau_{k}<t$ for all $k$ and $\tau_{k} \uparrow t$, it follows that $g(t-)=$ $h(t-)$.

(ii) We have $w(\hat{t}) \leq w(t)=u(t)-r=u(\hat{t})-r$. It follows that $w(\hat{t})=w(t), M(u, t)=$ $\{t\} \cup M(u, \hat{t})$ and $M(u, \tau)=M(u, \hat{t})$ for all $\tau \in(\hat{t}, t)$. Thus $g$ is constant on $[\hat{t}, t)$ and $g(t)=$ $\max \{h(t-), h(t), g(\hat{t})\}$ no matter whether the second or the third case in (4.18) applies.

(iii) We repeatedly use (3.18). We have $M(u, \tau) \subset M(u, \sigma)$ for all $\tau \leq \sigma$ with $\tau, \sigma \in\left[\tau_{1}, t\right]$. Thus, $g$ is nondecreasing on $\left[\tau_{1}, t\right]$. Moreover, $h\left(\tau_{k}\right) \leq g\left(\tau_{k}\right) \leq g(t)$ for all $k$. It follows that $h(t-) \leq g(t-) \leq g(t)$. We also have

$$
g(t)=\max \left\{g\left(\tau_{k}\right), \hat{g}_{k}\right\}, \quad \hat{g}_{k}:=\max \left\{\hat{h}(s): s \in\left(\tau_{k}, t\right] \cap M(u, t)\right\} .
$$

Since $\hat{g}_{k} \rightarrow \max \{h(t), h(t-)\}$ as $k \rightarrow \infty$, we have that (5.9) follows.

The right limit of $g$ at a boundary point $t$ behaves as follows.

Lemma 5.4. Let $t \in J \cap I_{+}, t<t^{*}$. Then $g(t+)$ exists and the following three cases arise.

(i) Let $u<u(t)$ on some interval $(t, t+\varepsilon)$. Then $g$ is constant on $[t, t+\varepsilon)$, in particular $g(t+)=$ $g(t)$.

(ii) Let $F_{\tau_{k}}(u)=u\left(\tau_{k}\right)=u(t)$ for some sequence $\tau_{k} \downarrow t$ with $\tau_{k}>t$ for all $k$. Then $g(t+)=$ $\max \{h(t+), g(t)\}$.

(iii) Let $F_{\tau}(u)>u(t)$ for all $\tau \in(t, t+\varepsilon)$, with some $\varepsilon>0$. Then $g(t+)=h(t+)$ and $w>w(t)$ on $(t, t+\varepsilon)$.

Proof. Again, we use Proposition 4.1. We have $u(t)-r=w(t)$ since $t \notin I_{0}$. If neither case (i) nor case (ii) arises, we have $u(t) \leq u\left(\tau_{k}\right)<F_{\tau_{k}}(u)$ for some sequence $\tau_{k} \downarrow t$; therefore $F_{\tau}(u)>u(t)$ in some interval $(t, t+\varepsilon)$ since $\tau \mapsto F_{\tau}(u)$ is nondecreasing.

In case (i), we have $M(u, \tau)=M(u, t)$ for all $\tau \in(t, t+\varepsilon)$, so $g=g(t)$ on $[t, t+\varepsilon)$.

In case (ii), we observe that $M(u, \tau) \subset M(u, \sigma)$ for all $\tau \leq \sigma$, with $\tau, \sigma \in\left[t, \tau_{1}\right]$. Thus, $g$ is nondecreasing on $\left[t, \tau_{1}\right]$. We have

$$
g\left(\tau_{k}\right)=\max \left\{g(t), \hat{g}_{k}\right\}, \quad \hat{g}_{k}:=\max \left\{\hat{h}(s): s \in\left(t, \tau_{k}\right] \cap M\left(u, \tau_{k}\right)\right\} .
$$

Since $\hat{g}_{k} \rightarrow h(t+)$ as $k \rightarrow \infty$, the claim follows.

In case (iii), we have, for all $\tau \in(t, t+\varepsilon)$,

$$
w(\tau) \geq F_{\tau}(u)-r>u(t)-r=w(t) \geq w\left(t_{*}\right)
$$


and $M(u, \tau) \subset(t, \tau]$. Thus, $g(\tau)=F_{\tau}^{\prime}(u ; h) \rightarrow h(t+)$ for $\tau \downarrow t$.

Lemma 5.5. Let $h$ be constant on an interval $J_{*}=\left[\tau_{*}, \tau^{*}\right] \subset J$. Then $g$ has at most one discontinuity point $t$ in $\left(\tau_{*}, \tau^{*}\right)$. Moreover, $g=h$ on $\left(t, \tau^{*}\right)$ if $t$ is such a point.

Proof. If $J_{*} \subset I_{0}$, then $g$ is constant on $J_{*}$ by Lemma 5.1. Otherwise, $J_{*}$ is a plus interval. Let $h=h_{*} \in \mathbb{R}$ in $J_{*}$. It follows from Proposition 4.1 and Corollary 3.1, applied to $J_{*}$ in place of $J$, that the only possible values for $g$ in $J_{*}$ are $g\left(\tau_{*}\right)$ and $h_{*}$. Therefore and since $g$ has right and left limits at all points of $J_{*}, g$ has only finitely many discontinuities in $J_{*}$ and is constant between discontinuities. Let $g$ be discontinuous at $t \in\left(\tau_{*}, \tau^{*}\right)$. Then $t \in I_{+}$by Lemma 5.1. It follows from Lemma 5.3 that $g(t)=h_{*}$ or $g(t-)=g(t) \neq g(t+)$; in both cases $g(t+)=h_{*}$ by Lemma 5.4. There can be no discontinuity $s \in\left(t, \tau^{*}\right)$ of $g$, since the smallest such $s$ would have to satisfy $h_{*}=g(t+)=g(s-)=g(s) \neq g(s+)$ by Lemma 5.3, a contradiction.

We next relate the function $g$ to the critical cone $K$ defined in (2.4). Since $J$ is a plus interval, we have $z>-r$ on $J$. Therefore, for $t \in J$, we have

$$
K(t)= \begin{cases}\mathbb{R}, & t \in A_{1}, \\ \mathbb{R}_{-}, & t \in A_{2}, \\ \{0\}, & t \in A_{3},\end{cases}
$$

where $A_{1}, A_{2}, A_{3}$ are given by (2.3).

Lemma 5.6. Let $t \in\left(t_{*}, t^{*}\right]$ or $t=t_{*}=a$. Then $(g(t+)-g(t-)) v \leq 0$ for all $v \in K(t)$.

Proof. If $t \in A_{1}$, we have $g(t+)=g(t)=g(t-)$ by Lemma 5.1. If $t \in A_{2}$, we have $g(t) \geq g(t-)$ by Lemma 5.3 and $g(t+) \geq g(t)$ by Lemma 5.4(i),(ii); recall that $g(a-)=g(a)$ and $g(b+)=$ $g(b)$ by convention. If $t \in A_{3}$, we have $v=0$.

Lemma 5.7. Let $h$ be right-continuous at $t \in J$. Then $h(t)-g(t+) \in K(t)$.

Proof. For $t \in A_{1}$, there is nothing to prove. For $t \in A_{3}$, we have $t<t^{*}$, and $h(t)-g(t+)=$ $h(t+)-g(t+)=0 \in K(t)$ by Lemma 5.4(iii). Let now $t \in A_{2}, t<t^{*}$. In case (ii) of Lemma 5.4, we have $h(t)-g(t+)=h(t+)-g(t+) \leq 0 \in K(t)$. In case (i) of Lemma 5.4, we have $h(t)-g(t+)=h(t)-g(t) \leq 0 \in K(t)$ since $t \in M(u, t)$. Finally, if $t=t^{*} \in A_{2}$, we have $t=b$ since $\Delta$ is regular, $K(b)=\mathbb{R}_{-}$and $g(b+)=g(b) \geq h(b)$ by Lemma 5.3.

We define $\gamma_{h}:[a, b] \rightarrow \mathbb{R}$ by

$$
\gamma_{h}(t)=(h(t)-g(t+))(g(t+)-g(t-)), \quad t \in[a, b] .
$$

Lemma 5.8. Let $h$ be right-continuous at $t \in J, t \neq a$. Then $\gamma_{h}(t)=0$ or

$$
\gamma_{h}(t)=(h(t)-h(t-))(h(t-)-g(t-)), \quad g(t)=h(t-) .
$$

We also have $\gamma_{h}(a)=0$.

Proof. Let $\gamma_{h}(t) \neq 0$. Then $g(t+) \neq g(t-)$ and therefore $t \notin I_{0}$ by Lemma 5.1. Moreover, $h(t+)=h(t) \neq g(t+)$, so $g(t)=g(t+) \neq h(t)$ by Lemma 5.4 if $t \neq b$; also, $g(b)=g(b+)$ by definition. In addition, $g(t)-g(t-)=g(t+)-g(t-) \neq 0$. Since $g(t)=\max \{h(t), h(t-), g(t-)\}$ by Lemma 5.3, it follows that $g(t)=h(t-)$. That $\gamma_{h}(a)=0$ is a direct consequence of Lemma 5.4 applied with $t=a$. 
Now we consider the behaviour of $g$ on the whole interval $[a, b]$.

Lemma 5.9. The function $g$ is constant on every interval contained in $I_{0}$.

Proof. By Lemma 5.1, $g$ is constant on intervals $I_{J}$ contained in $I_{0} \cap J$. By Proposition 4.2, this also applies if $J$ is a minus interval. Concatenating such intervals yields the result for arbitrary intervals contained in $I_{0}$.

Lemma 5.10. Let $g$ be discontinuous at $t \in(a, b)$. Then

$$
g(t+) \in\{h(t-), h(t), h(t+)\} .
$$

If moreover $h$ is continuous at $t$, then $g(t) \in\{g(t+), g(t-)\}$.

Proof. We have $t \notin I_{0}$ by Lemma 5.9 and, since $\Delta$ is regular, $t \in \operatorname{int}(J)$ for some partition interval of $\Delta$. Let $J$ be a plus interval for $\left(u, z_{0}\right)$. Then $g(t+)=h(t+)$ or $g(t+)=g(t)$ by Lemma 5.4; in the latter case, $g(t) \neq g(t-)$ and hence $g(t)=h(t)$ or $g(t)=h(t-)$ by Lemma 5.3. Thus (5.13) holds. If $h$ is continuous at $t$, then $g(t)=\max \{h(t), g(t-)\}$ by Lemma 5.3 and therefore $g(t)=g(t-)$ or $g(t)=h(t)$; in the latter case, $g(t+)=g(t)$ by Lemma 5.4.

If $J$ is a minus interval for $\left(u, z_{0}\right)$, it is a plus interval for $\left(-u,-z_{0}\right)$ and

$$
g(t)=-\mathscr{P}_{r, t}^{\prime}\left(\left[-u ;-z_{0}\right] ;\left[-h,-y_{0}\right]\right)
$$

by Proposition 4.2. Applying what we just have shown for plus intervals completes the proof.

We say that $f:[a, b] \rightarrow \mathbb{R}$ is a step function if the set of its values and the set of its discontinuity points are finite. (Thus, a step function is constant between successive discontinuity points.)

Lemma 5.11. Let $\left(u, z_{0}\right) \in C[a, b] \times \mathbb{R}, y_{0} \in \mathbb{R}$, and let $h:[a, b] \rightarrow \mathbb{R}$ be a step function. Then $g$ is a step function, and

$$
\operatorname{var}(g) \leq \operatorname{var}(h)+\left|y_{0}\right|
$$

Proof. In order to prove that $g$ is a step function, it suffices to prove that $g \mid J$ is a step function for every partition interval $J$ of $\Delta$. If $J$ is a plus interval for $\left(u, z_{0}\right)$, this follows immediately from Lemma 5.5. If $J$ is a minus interval for $\left(u, z_{0}\right)$, we pass to $\left(-u,-z_{0}\right)$ and $\left(-h,-y_{0}\right)$. Since $-h$ is a step function, in view of Proposition 4.2 the claim again follows from Lemma 5.5.

Let us now prove (5.14). Let $t_{1}<\cdots<t_{N-1}$ be the discontinuity points of $g$ in $(a, b)$, and let $t_{0}=a$ and $t_{N}=b$. (That is, $N=1$ if $g$ is constant on $(a, b)$.) We have

$$
\operatorname{var}(g)=|g(a+)-g(a)|+\sum_{k=1}^{N}\left(\left|g\left(t_{k}+\right)-g\left(t_{k}\right)\right|+\left|g\left(t_{k}\right)-g\left(t_{k-1}+\right)\right|\right) .
$$

By

$$
g_{0}, g_{1}, g_{2}, \ldots, g_{2 N}, g_{2 N+1}
$$

we denote the finite sequence $g(a), g(a+), g\left(t_{1}\right), \ldots, g\left(t_{N-1}+\right), g(b)$. We define a partition $0=$ $i_{0}<\ldots i_{M}=2 N+1$ of $0, \ldots, 2 N+1$ by

$$
i_{j}=\left\{\begin{array}{ll}
\min B_{j}, & B_{j} \neq \emptyset, \\
2 N+1, & B_{j}=\emptyset,
\end{array}, \quad B_{j}:=\left\{i: i_{j-1}<i \leq 2 N+1, g_{i_{j}} \neq g_{i_{j-1}}\right\}\right.
$$


Thus, $g_{i}$ is constant on $\left[i_{j-1}, i_{j}-1\right]$ resp. $\left[i_{j-1}, 2 N+1\right]$. It follows from (5.15) that

$$
\operatorname{var}(g)=\sum_{j=1}^{M}\left|g_{i_{j}}-g_{i_{j-1}}\right| .
$$

Since $g$ is locally constant on $I_{0}$, by definition of a regular partition, each point $t_{k} \neq b$ belongs to the interior of some interval $J$ of $\Delta$. If $J$ is a plus interval, by Lemmas 5.3 and 5.4 and by (4.16), we have

$$
\begin{array}{ll}
g_{i_{j}}=h\left(t_{k}+\right), & \text { if } g_{i_{j}} \text { corresponds to } g\left(t_{k}+\right), \\
g_{i_{j}}=h\left(t_{k}\right) \text { or } g_{i_{j}}=h\left(t_{k}-\right), & \text { if } g_{i_{j}} \text { corresponds to } g\left(t_{k}\right), \\
g_{i_{0}}=h(a)-\pi_{Z}^{\prime}\left(z_{0} ; y_{0}\right), & \text { and }\left|\pi_{Z}^{\prime}\left(z_{0} ; y_{0}\right)\right| \leq\left|y_{0}\right| .
\end{array}
$$

If $J_{i}$ is a minus interval, the same is true due to (4.19). From (5.16), we conclude that $\operatorname{var}(g) \leq$ $\operatorname{var}(h)+\left|y_{0}\right|$.

Lemma 5.12. Let $\left(u, z_{0}\right) \in C[a, b] \times \mathbb{R}, y_{0} \in \mathbb{R}$, and let $h:[a, b] \rightarrow \mathbb{R}$ be a right-continuous step function. Then

$$
\sum_{t \in[a, b]}\left|\gamma_{h}(t)\right| \leq\left(\operatorname{var}(h)+\left|y_{0}\right|\right) \cdot \max _{t \in[a, b]}|h(t)-h(t-)|
$$

Proof. Since $g$ is a step function by Lemma 5.11, the set $\Gamma=\left\{\gamma_{h} \neq 0\right\}$ is finite. Let $t_{1}<\cdots<t_{N}$ be the elements of $\Gamma$. The assertions of Lemma 5.8 hold on minus intervals of $\Delta$ as well as on plus intervals, because $g$ and $h$ both change sign by passing over to $\left(-u,-z_{0}\right)$ and $\left(-h,-y_{0}\right)$. Thus, $t_{1}>a$ and

$$
\gamma_{h}\left(t_{k}\right)=\left(h\left(t_{k}\right)-h\left(t_{k}-\right)\right)\left(h\left(t_{k}-\right)-g\left(t_{k}-\right)\right), \quad 1 \leq k \leq N,
$$

by Lemma 5.8. Since $g\left(t_{1}-\right)=g(a)=h(a)-\pi_{Z}^{\prime}\left(z_{0} ; y_{0}\right)$, we conclude from (4.16) that

$$
\left|\gamma_{h}\left(t_{1}\right)\right| \leq\left|h\left(t_{1}\right)-h\left(t_{1}-\right)\right|\left(\left|h\left(t_{1}-\right)-h(a)\right|+\left|y_{0}\right|\right) .
$$

For $k>1$, we have $g\left(t_{k}-\right)=g\left(t_{k-1}+\right)$. Since $t_{k-1} \in \Gamma$, we obtain from (5.18) and Lemma 5.10 that

$$
\gamma_{h}\left(t_{k}\right)=\left(h\left(t_{k}\right)-h\left(t_{k}-\right)\right)\left(h\left(t_{k}-\right)-\tilde{h}_{k-1}\right)
$$

with $\tilde{h}_{k-1} \in\left\{h\left(t_{k-1}+\right), h\left(t_{k-1}\right), h\left(t_{k-1}-\right)\right\}$. It follows that by setting $\tilde{h}_{0}=h(a)$

$$
\begin{aligned}
\sum_{t \in[a, b]}\left|\gamma_{h}(t)\right| & =\left|y_{0}\right| \cdot\left|h\left(t_{1}\right)-h\left(t_{1}-\right)\right|+\sum_{k=1}^{N}\left|h\left(t_{k}-\right)-\tilde{h}_{k-1}\right| \cdot\left|h\left(t_{k}\right)-h\left(t_{k}-\right)\right| \\
& \leq\left(\operatorname{var}(h)+\left|y_{0}\right|\right) \cdot \max _{t \in[a, b]}|h(t)-h(t-)| .
\end{aligned}
$$

Lemma 5.13. Let $\left(u, z_{0}\right) \in C[a, b] \times \mathbb{R},\left(h, y_{0}\right) \in G[a, b] \times \mathbb{R}$. Then the following holds:

(i) We have $(g(t+)-g(t-)) v \leq 0$ for all $t \in[a, b]$ and all $v \in K(t)$.

(ii) Let $t \in[a, b]$ with $h$ right-continuous at $t$. Then $h(t)-g(t+) \in K(t)$.

Proof. On each interval $J$ of $\Delta$, we apply Lemma 5.6 and Lemma 5.7, either directly if $J$ is a plus interval for $\left(u, z_{0}\right)$, or after passing to $\left(-u,-z_{0}\right)$ and $\left(-h,-y_{0}\right)$ if $J$ is a minus interval, using Proposition 4.2 again. Note that the boundary points of $J$ which are different from $a$ and $b$ belong to $A_{1}$. 
We recall that $g_{+}, g_{-}:[a, b] \rightarrow \mathbb{R}$ denote the right and left limit $g_{+}(t)=g(t+)$ and $g_{-}(t)=$ $g(t-)$ of $g$.

Proposition 5.1. Let $u \in C[a, b], h \in C[a, b] \cap B V[a, b], z_{0}, y_{0} \in \mathbb{R}$. Then the pointwise directional derivative $g$ from (5.1) belongs to $B V_{r l}[a, b]$, satisfies $\operatorname{var}(g) \leq \operatorname{var}(h)+\left|y_{0}\right|$ as well as $g(t) \in$ $\{g(t+), g(t-)\}$ for all $t \in[a, b]$, and solves the system

$$
\begin{gathered}
g(a)=h(a)-\pi_{Z}^{\prime}\left(z_{0} ; y_{0}\right), \\
h(t)-g_{+}(t) \in K(t), \quad \text { for all } t \in[a, b], \\
\int_{a}^{b}\left(h(t)-g_{+}(t)-v(t)\right) d g(t) \geq 0
\end{gathered}
$$

for all $v \in G[a, b]$ with $v(t) \in K(t)$ for all $t \in[a, b]$.

Proof. Since $h \in C[a, b] \cap B V[a, b]$, there exist right-continuous step functions $h_{n}:[a, b] \rightarrow \mathbb{R}$ with $h_{n} \rightarrow h$ uniformly and $\operatorname{var}\left(h_{n}\right) \leq \operatorname{var}(h)$ for all $n$. We define $g_{n}:[a, b] \rightarrow \mathbb{R}$ by $g_{n}(t)=$ $\mathscr{P}_{r, t}^{\prime}\left(\left[u ; z_{0}\right] ;\left[h_{n}, y_{0}\right]\right)$. By Lemma 5.11, $g_{n}$ is a step function with $\operatorname{var}\left(g_{n}\right) \leq \operatorname{var}\left(h_{n}\right)+\left|y_{0}\right| \leq$ $\operatorname{var}(h)+\left|y_{0}\right|$. By the Lipschitz estimate (4.20), $g_{n} \rightarrow g$ uniformly and thus $g \in B V[a, b]$ with $\operatorname{var}(g) \leq \operatorname{var}(h)+\left|y_{0}\right|$. Moreover, $g(t) \in\{g(t+), g(t-)\}$ for all $t \in[a, b]$ by Lemma 5.10 since we assume that $h \in C[a, b]$. For all $n$, we have $g_{n}(a)=h_{n}(a)-\pi_{Z}^{\prime}\left(z_{0} ; y_{0}\right)$, and $h_{n}(t)-g_{n}(t+) \in$ $K(t)$ for all $t \in[a, b]$ due to Lemma 5.13(ii). Passing to the limit $n \rightarrow \infty$ yields (5.21) and (5.22).

In order to prove (5.23), let $n$ be fixed and let $t_{1}<\cdots<t_{N}$ be the discontinuity points of $g_{n}$ in $(a, b)$ (set $N=0$ if there are no such points) and set $t_{N+1}=b$. Let $\left\{s_{-1}, \ldots, s_{N+1}\right\}$ be a partition of $[a, b]$ of the form

$$
a=t_{0}=s_{-1}<s_{0}<t_{1}<s_{1}<\cdots<s_{N-1}<t_{N}<s_{N}<s_{N+1}=t_{N+1}=b .
$$

Since $g_{n}$ is a step function, $g_{n}=g_{n}\left(t_{k}-\right)$ on $\left[s_{k-1}, t_{k}\right)$ and $g_{n}=g_{n}\left(t_{k}+\right)$ on $\left(t_{k}+, s_{k}\right]$ for $1 \leq k \leq N$. Let $v \in G[a, b]$ with $v(t) \in K(t)$ for all $t \in[a, b]$. Then, for every $k \in\{0, \ldots, N+1\}$, we have from (6.2) that

$$
\begin{aligned}
\int_{s_{k-1}}^{s_{k}}\left(h_{n}(t)\right. & \left.-g_{n}(t+)-v(t)\right) d g_{n}(t) \\
= & \left(h_{n}\left(t_{k}\right)-g_{n}\left(t_{k}+\right)-v\left(t_{k}\right)\right)\left(g_{n}\left(t_{k}+\right)-g_{n}\left(t_{k}-\right)\right) .
\end{aligned}
$$

Using Lemma 5.12, we obtain the estimate

$$
\begin{gathered}
\int_{a}^{b}\left(h_{n}(t)-g_{n}(t+)\right) d g_{n}(t)=\sum_{k=0}^{N+1}\left(h_{n}\left(t_{k}\right)-g_{n}\left(t_{k}+\right)\right)\left(g_{n}\left(t_{k}+\right)-g_{n}\left(t_{k}-\right)\right) \\
=\sum_{k=0}^{N+1} \gamma_{h_{n}}\left(t_{k}\right) \geq-\left(\operatorname{var}\left(h_{n}\right)+\left|y_{0}\right|\right) \cdot \max _{t \in[a, b]}\left|h_{n}(t)-h_{n}(t-)\right| .
\end{gathered}
$$

Moreover, $\operatorname{var}\left(g_{n}\right) \leq \operatorname{var}\left(h_{n}\right)+\left|y_{0}\right| \leq \operatorname{var}(h)+\left|y_{0}\right|$ by Lemma 5.11. Due to Proposition 6.3, we may pass to the limit $n \rightarrow \infty$ on the left side of (5.25). Since $\operatorname{var}\left(h_{n}\right) \leq \operatorname{var}(h)$ and $h$ is continuous, the right side of (5.25) converges to 0 as $n \rightarrow \infty$. Moreover,

$$
\int_{a}^{b}-v(t) d g_{n}(t)=\sum_{k=1}^{N+1}\left(-v\left(t_{k}\right)\right)\left(g_{n}\left(t_{k}+\right)-g_{n}\left(t_{k}-\right)\right) \geq 0
$$


by Lemma 5.13(i). It follows from Proposition 6.3 that

$$
\int_{a}^{b}-v(t) d g(t) \geq 0
$$

Thus (5.23) holds and the proof is complete.

From the variational integral over $[a, b]$ in (5.23), we can obtain a variational integral over $[a, s]$ for all $s \in(a, b)$ as required in Theorem 2.1 .

Proposition 5.2. In the situation of Proposition 5.1, moreover

$$
\int_{a}^{s}\left(h(t)-g_{+}(t)-v(t)\right) d g(t) \geq 0
$$

holds for all $s \in(a, b]$ and all $v \in G_{K}[a, s]$, where

$$
G_{K}[a, s]=\{v: v \in G[a, s], v(t) \in K(t) \text { for all } t \in[a, s]\} .
$$

Proof. The proof is based on the choice of suitable test functions in (5.23). For $s=b$, there is nothing left to prove. Let $s \in(a, b)$ and $v \in G_{K}[a, s]$ be given. We have already proved in Proposition 5.1 that $g(s)=g_{-}(s)$ or $g(s)=g_{+}(s)$. In the first case, we set

$$
\tilde{v}=v \chi_{[a, s)}+\left(h-g_{+}\right) \chi_{[s, b]} .
$$

Here and below, for $A \subset[a, b]$ by $\chi_{A}$ we denote the characteristic function of $A$ that is 1 on $A$ and 0 elsewhere.

By (5.22), $\tilde{v}$ is an admissible test function in (5.23). By (5.23) and since $h-g_{+}-\tilde{v}=0$ on $[s, b]$, we have from Proposition 6.1 and (6.1) that

$$
\begin{aligned}
0 & \leq \int_{a}^{b}\left(h-g_{+}-\tilde{v}\right) d g=\int_{a}^{s}\left(h-g_{+}-\tilde{v}\right) d g \\
& =\int_{a}^{s}\left(h-g_{+}-v\right) d g+\int_{a}^{s}(v-\tilde{v}) \chi_{\{s\}} d g \\
& =\int_{a}^{s}\left(h-g_{+}-v\right) d g+(v(s)-\tilde{v}(s))(g(s)-g(s-)) .
\end{aligned}
$$

Thus (5.26) holds due to $g(s)=g(s-)$. In the case $g(s)=g(s+)$, we choose

$$
\tilde{v}=v \chi_{[a, s]}+\left(h-g_{+}\right) \chi_{(s, b]} .
$$

Again, $\tilde{v}$ is an admissible test function in (5.23). It follows from (5.23) that

$$
0 \leq \int_{a}^{s}\left(h-g_{+}-v\right) d g+\int_{s}^{b}\left(h-g_{+}-\tilde{v}\right) d g .
$$

Using Proposition 6.1 and (6.1), we get

$$
\int_{s}^{b}\left(h-g_{+}-\tilde{v}\right) d g=\int_{s}^{b}\left(h-g_{+}-v\right) \chi_{\{s\}} d g=\left(h-g_{+}-v\right)(s)(g(s+)-g(s))=0 .
$$

Again, (5.26) holds and the proof is complete. 


\section{APPENDIX: RESULTS FROM INTEGRATION THEORY}

For the definition and properties of the Kurzweil-Stieltjes integral of functions $f, g:[a, b] \rightarrow$ $\mathbb{R}$, denoted by

$$
\int_{a}^{b} f d g \quad \text { or } \quad \int_{a}^{b} f(t) d g(t)
$$

we refer to [10]. In particular, it is defined if $f \in G[a, b]$ and $g \in B V[a, b]$, see [10, Theorem 6.3.11]. The mappings $f \mapsto \int_{a}^{b} f d g$ and $g \mapsto \int_{a}^{b} f d g$ are linear.

We present some specific formulas and results which we use in the paper. The definitions $g(a-)=g(a)$ and $g(b+)=g(b)$ always apply.

For $f:[a, b] \rightarrow \mathbb{R}, g \in G[a, b]$ and $\tau \in[a, b]$, we have

$$
\int_{a}^{b} f \chi_{\{\tau\}} d g=f(\tau) \int_{a}^{b} \chi_{\{\tau\}} d g=f(\tau)(g(\tau+)-g(\tau-)) .
$$

If $f, g:[a, b] \rightarrow \mathbb{R}, \tau \in[a, b]$ and $g$ is constant on $[a, \tau)$ as well as on $(\tau, b]$, then

$$
\int_{a}^{b} f d g=f(\tau)(g(\tau+)-g(\tau-))
$$

Both (6.1) and (6.2) follow from the formulas in Lemma 6.3.2 and Lemma 6.3.3 in [10].

Proposition 6.1. Let $f \in G[a, b], g \in B V[a, b], a<s<b$. Then

$$
\int_{a}^{b} f d g=\int_{a}^{s} f d g+\int_{s}^{b} f d g
$$

Proof. See Theorem 6.2.10 in [10].

Proposition 6.2. Let $g \in B V[a, b]$. Then

$$
\int_{a}^{b} g_{+} d g=\frac{1}{2}\left(g(b)^{2}-g(a)^{2}\right)+\frac{1}{2} \sum_{a \leq t \leq b}(g(t+)-g(t-))^{2} .
$$

Proof. This is a special case of Corollary 1.13 in [12]. It can also be derived from Proposition 6.4.2 and the second formula of Lemma 6.3.16 in [10].

Proposition 6.3. Let $\left\{f_{n}\right\} \subset G[a, b],\left\{g_{n}\right\} \subset B V[a, b]$ with $\sup _{n} \operatorname{var}\left(g_{n}\right)<\infty$ and $f_{n} \rightarrow f$ as well as $g_{n} \rightarrow g$ uniformly for $n \rightarrow \infty$. Then $f \in G[a, b], g \in B V[a, b]$ and

$$
\lim _{n \rightarrow \infty} \int_{a}^{b} f_{n} d g_{n}=\int_{a}^{b} f d g
$$

Proof. This is a special case of Theorem 6.8.8 in [10].

\section{Acknowledgements}

This research was supported by the GAČR Grant 20-14736S, and by the European Regional Development Fund, Project No. CZ.02.1.01/0.0/16_019/0000778. 


\section{REFERENCES}

[1] M. Brokate, J. Sprekels, Hysteresis and Phase Transitions, Springer, 1996.

[2] M. A. Krasnoselskii, A. V. Pokrovskii, Systems with Hysteresis, Springer, 1989.

[3] P. Krejčí, Hysteresis, Convexity and Dissipation in Hyperbolic Equations, Gakkōtosho, Tokyo 1996.

[4] P. Krejčí, Evolution variational inequalities and multidimensional hysteresis operators. In: Nonlinear Differential Equations, (P. Drábek, P. Krejčí, P. Takáč eds.), Research Notes in Mathematics, Vol. 404, pp. 47-110, Chapman \& Hall/CRC, London, 1999.

[5] I. Mayergoyz, Mathematical Models of Hysteresis, Springer 1991.

[6] A. Mielke, T. Roubíček, Rate-independent Systems, Springer 2015.

[7] J.-J. Moreau, Evolution problem associated with a moving convex set in a Hilbert space, J. Differential Equations 26 (1977), 347-374.

[8] A. Visintin, Differential Models of Hysteresis, Springer, Berlin, 1994.

[9] M. Brokate, P. Krejčí, Weak differentiability of scalar hysteresis operators, Discrete Continuous Dyn. Syst. Ser. A 35 (2015), 2405-2421.

[10] G.A. Monteiro, A. Slavík, M. Tvrdý, Kurzweil-Stieltjes Integral, World Scientific, Singapore 2015.

[11] P. Krejčí: The Kurzweil integral and hysteresis, J. Phys. Conf. Ser. 55 (2006), 144-154.

[12] P. Krejčí, M. Liero, Rate independent Kurzweil processes, Appl. Math. 54 (2009), 117-145.

[13] A. Alphonse, M. Hintermüller, C.N. Rautenberg: Directional differentiability for elliptic quasi-variational inequalities of obstacle type. Calc. Var. 58, 39 (2019). https://doi.org/10.1007/s00526-018-1473-0.

[14] C. Christof, Sensitivity analysis of elliptic variational inequalities of the first and the second kind, PhD Thesis, Fakultät für Mathematik, TU Dortmund, 2018.

[15] C. Christof, Sensitivity analysis and optimal control of obstacle-type evolution variational inequalities, SIAM J. Control Optim. 57 (2019), 192-218.

[16] J. Jarušek, M. Krbec, M. Rao, J. Sokołowski, Conical differentiability for evolution variational inequalities, J. Differential Equations 193 (2003), 131-146.

[17] F. Mignot, Contrôle dans les inéquations variationelles elliptiques, J. Funct. Anal. 22 (1976), 130-185.

[18] J. Sokolovski, J.-P. Zolésio, Introduction to Shape Optimization, Springer, New York, 1992.

[19] G. Wachsmuth, A guided tour of polyhedric sets: basic properties, new results on intersections and applications, J. Convex Anal. 26 (2019), 153-188.

[20] G. Wachsmuth, Elliptic quasi-variational inequalities under a smallness assumption: uniqueness, differential stability and optimal control, Calc. Var. 59 (2020), 82.

[21] M. Brokate, Newton and Bouligand derivatives of the scalar play and stop operator, Math. Model. Nat. Phenom. 15 (2020), 51.

[22] I.V. Girsanov, Lectures on Mathematical Theory of Extremum Problems, Springer, Berlin, 1972.

[23] D. Fraňková, Regulated functions, Math. Bohem. 116 (1991), 20-59.

[24] A. Mielke, R. Rossi, G. Savaré, Modeling solutions with jumps for rate-independent systems on metric spaces, Discrete Contin. Dyn. Syst. 25 (2009), 585-615. 